\title{
Incidence of Methicillin-Resistant Staphylococcus aureus (MRSA) In a Small Poultry in South West, Nigeria.
}

\author{
Adeyeye J. OKE ${ }^{1}$, Adewale A. OKE ${ }^{2}$ \\ ${ }^{1}$ (Department of Medical Microbiology, Faculty of Basic Clinical Sciences, Bowen University Teaching \\ Hospital, Ogbomoso. Nigeria). \\ ${ }^{2}$ (Department of Biological Sciences, College Of Natural Sciences, Redeemer's University, Mowe. Nigeria)
}

\begin{abstract}
Microbiological studies were carried out in a small poultry farm in a Community in South West Nigeria following a report of acute diarrhea in 6-week old broilers, catarrh and cough in the poultry attendants. Nasal swabs were collected from 25 symptomatic and 5 asymptomatic attendants. Swabs were collected from the cloacae of 100 birds with diarrhea. Twenty-five (100\%) symptomatic attendants carried Methicillin-resistant Staphylococcus aureus (MRSA) in their nostrils. Five asymptomatic attendants carried Methicillin-sensitive Staphylococcus aureus (MSSA). Ninety five (95\%) cloacae swabs yielded MRSA and five (5\%) yielded Lactosefermenting (LF) isolates suggestive of Escherichia coli on MacConkey agar. There was an incidence of $83.3 \%$ MRSA in the poultry attendants, and 95\% in chickens in the poultry. Cross infection of MRSA seems to occur from chickens to the attendants in the poultry farm.
\end{abstract}

Keywords: Chickens, MRSA, Poultry attendants

\section{Introduction}

Methicillin-resistant Staphylococcus aureus (MRSA) has been detected in several species and animalderived products (1). MRSA was found in milk from Belgium cow with mastitis in 1972. The MRSA status of the dairymen was not investigated (2). MRSA has since been reported in many diverse species including $\operatorname{dogs}(3,4) \operatorname{cats}(5,6,7)$; psittacine birds(7,8), chickens(9). MRSA infections in companion animals involved strains resembling nosocomial strains including epidemic MRSA (EMRSA). When epidemic Hospital associated MRSA (HA-MRSA) clones were observed in dogs, the assumption was that the direction of spread has been from man to animals. However, strains of MRSA that are thought to have evolved in animals colonize and infect human attendants (10).

Several workers have reported Staphylococcus aureus infections in poultry: Eric et al (2001) reported that staphylococcus infections tend to occur more frequently during four periods: 0-2, 4-6, 10-20 and 24-30 weeks old, manifesting in omphalitis, femoral necrosis, coccidiosis, infected hock, stifle joints, planter abscesses (11). Capital et al (2001) reported isolation of Staphylococcus aureus from retail chicken carcasses in Spain (12). Kohler et al (1980) reported that Staphylococcus aureus was found to be the most important pathogen of arthritis with synovitis and of osteomyelitis (13). Andrew et al (2011) also reported 52\% occurrence of multidrug-resistant Staphylococcus aureus in the US meat and poultry (14).

The extensive use of antimicrobial agents in food animals' production, where they are often applied subtherapeutically for growth production and routine disease prevention, often give rise to Multidrug-resistant and MRSA strains in poultry (15). Poultry producers commonly use antibiotics to promote growth of the chicken. This can lead to bacteria in the chicken's digestive systems becoming resistant to antibiotics (16).

The aim of this study was to isolate the causative agents of the reported acute upper respiratory tract infection in poultry attendants, and diarrhea in the chickens in a small poultry. Our focus was on bacteria agents. The antibiotic used prophylactically and therapeutically in the farm include: oxytetracycline, ampicillin, sulphonamide and metronidazole.

\subsection{Materials}

\section{Materials and Methods}

Sterile swab sticks (Sterilin Ltd UK)

Petri dishes-plastic (Sterilin Ltd UK)

Brain heart infusion broth (LAB M Ltd., Lanchester. UK)

Mannitol salt agar (Oxoid Ltd.UK)

MacConkey agar (Biotec Medical Market Ltd., Middlesex.UK)

Nutrient agar (Biotec Medical Market Ltd., Middlesex.UK)

Muller Hinton agar (Biotec Medical Market Ltd., Middlesex.UK)

Antibiotic sensitivity Discs (ABTEX Biological Ltd, Liverpool.UK) 
Blood agar [Prepared by dissolving $28 \mathrm{~g}$ Nutrient agar in 1Litre distilled water, autoclaved $\left(121{ }^{\circ} \mathrm{C}\right.$ for 15 minutes), cooled $\left(40-45^{\circ} \mathrm{C}\right), 25 \mathrm{ml}$ of sterile defibrinated blood added aseptically, mixed thoroughly and poured into new sterile plastic petri dishes inside a clean safety hood. Quality control (Sterility check was done on the prepared blood agar plates].

\subsection{Methods} workers.

Official consent was obtained from the Director of the poultry and informed consent from the poultry

Nasal swabs were taken from all the thirty (30) volunteered poultry attendants having constant, frequent contacts with the chickens. Twenty-five (25) of them had symptoms of catarrh and cough, and five showing no such symptoms. Paired cloacae swabs were taken from one hundred (100) six-week old chickens. The swabs were first incubated in Brain heart infusion broth (as described by Persoons et al (2009) but without antibiotic supplements. After overnight incubation at $37^{\circ} \mathrm{C}, 1 \mu \mathrm{L}$ of the broth was streaked onto Mannitol salt agar (MSA), MacConkey agar (McC), Blood agar (BA), and incubated for 48 hours at $37^{\circ} \mathrm{C}$. The other pairs of cloacae swabs were examined for ova of parasites. Growth on MSA showing characteristics of Staphylococcus aureus were sub-cultured on $\mathrm{McC}$ and incubated for 24 hours at $37^{\circ} \mathrm{C}$. Slide and tube Coagulase and calatase tests were done on the pure isolates. Lactose-fermenting growth on $\mathrm{McC}$ showing characteristics of Escherichia coli were tested for Indole and citrate.

Antibiotic sensitivity using Disc diffusion technique on Muller Hinton agar was done on all the Staphylococcus aureus isolates, using the following antibiotic discs: Erythromycin $(15 \mu \mathrm{g})$, Cloxacillin $(5 \mu \mathrm{g})$, Gentamicin $(10 \mu \mathrm{g})$, Augmentin $(30 \mu \mathrm{g})$, Cotrimoxazole $(25 \mu \mathrm{g})$, Amoxycillin $(30 \mu \mathrm{g})$, Tetracycline $(30 \mu \mathrm{g})$, Chloramphenicol $(30 \mu \mathrm{g})$. Multidrug-resistant Staphylococcus aureus were further screened with Oxacillin $(1 \mu \mathrm{g})$, Methicillin $(1 \mu \mathrm{g})$ Cefoxitin $(30 \mu \mathrm{g})$. Zone diameters of inhibition were measured in $\mathrm{mm}$.

All Staphylococcus aureus taken as MRSA had zone diameter of inhibition $0-5 \mathrm{~mm}$, using the clinical laboratory standards institute (CLSI guidelines (M31-A3)

\section{Results}

Ninety five percent (95\%) and 83.3\% MRSA incidences were recorded in chickens with diarrhea, and poultry attendants respectively. Seventeen percent (17\%) MSSA were from the asymptomatic attendants. Only $5 \%$ coliform, suspected to be $E$. coli was recorded. (Table 1a, 1b) The isolates, lactose-fermenter on McC, were pure, unmixed and were all positive for indole, negative for citrate tests. The cultures yielded pure, unmixed growth of Staphylococcus aureus on all the media used. No parasite was found in all the chickens.

TABLE 1a SHOWING FREQUENCY OF ISOLATION OF MRSA AND MSSA IN POULTRY ATTENDANTS ( $\mathbf{N}=\mathbf{3 0})$

\begin{tabular}{|l|c|c|}
\hline \multicolumn{1}{|c|}{ CATEGORY OF ATTENDANTS } & ORGANISMS ISOLATED & NUMBER OF ATTENDANTS \\
\hline Symptomatic attendants & MRSA & $25(83.3 \%)$ \\
\hline Asymptomatic attendants & MSSA & $5(16.7 \%)$ \\
\hline
\end{tabular}

TABLE 1b SHOWING FREQUENCY OF ISOLATION OF BACTERIA IN CHICKENS (n=100)

\begin{tabular}{|l|c|}
\hline \multicolumn{1}{|c|}{ BACTERIA ISOLATED } & NUMBERS OF CHICKEN \\
\hline MRSA & $95(95 \%)$ \\
\hline Coliform (suggestive of $E$. coli) & $5(5 \%)$ \\
\hline
\end{tabular}

\section{Discussion}

Ninety five percent (95\%) MRSA in the cloacae swabs, and that the cultures yielded pure, unmixed growth of Staphylococcus aureus on all the media used suggest a high prevalence and that MRSA may have been the causative agent of diarrhea in the Chickens. In the attendants none of them carrying MSSA had any symptoms, whereas symptoms of catarrh and cough occur in all of them who carried MRSA. Therefore MRSA may have been responsible for this.

Methicillin-resistant Staphylococcus aureus have been reported severally in meat and chicken $(3,5,9)$, but no report has linked the organism directly to diarrhea in chicken. However, $95 \%$ incidence of MRSA in chicken with diarrhea in this study may suggest the importance of MRSA in this condition, moreso that the isolates were pure, unmixed growth of Staphylococcus aureus. Further study is needed in understanding the role of MRSA in diarrhea in chicken.

Buckley (2012) reported that chicken-related staphylococcus infections are usually attributed to Staphylococcus aureus and that the organism can be passed to people via digestion of contaminated meat, and can also be transferred as a result of physical contact with live birds (17). Wickline (2013) reported a high prevalence of multidrug-resistant Staphylococcus aureus in the nostrils of industrial livestock workers (18). She 
also reported incidences of MRSA in the workers. Graham (2009) found staphylococcus and enterococcus in the digestive systems of both chickens and people. He also reported that antimicrobial-resistant bacteria are excreted and wind up in the poultry litters.

The high incidences of MRSA in chickens and attendants in this study suggest cross infection from chicken to the attendants having physical contact with the chickens and their faecal materials. Our attention was on the high prevalence of MRSA, so we did not work more on the insignificant number of E. coli.

\section{Acknowledgement}

The authors wish to acknowledge the Medical Director Shalom Medical Centre, Ogbomoso, Dr. Amao, for supplying consumables.

\section{REFERENCES}

[1]. Persoons D, Van Hoorebeke S, Hermans K, Butaye P, de Kruif A, Haesebrouck F, Dewulf J. 2009. Methicillin- resistant Staphylococcus aureus in Poultry. Emerg Infect Dis. 15(3):452-453. Erratum in 15(5):853.

[2]. Morgan M. 2008 Methicillin-resistant Staphylococcus aureus and animals: Zoonosis or humanosis? J Antimicrob Chemother. 62:1181-1187.

[3]. Cefai C, Ashurst S, Ownens C. 1994 Human carriage of Methicillin-resistant Staphylococcus aureus linked with a pet dog. Lancet 344:539-540.

[4]. Marian FA. 2003. Asymptomatic nasal carriage of mupirocin-resistant methicillin-resistant Staphylococcus aureus (MRSA) in a pet dog association with MRSA infection in household contacts. Clin infect Dis. 36: e26-e28.

[5]. O’Mahony R, Abbott Y, Leonard FC Markey BK, Quinn PJ, Pollock PJ, Fanning S, Rossney AS. 2005. Methicillin-resistant Staphylococcus aureus (MRSA) isolated form animals and Veterinary personnel in Ireland. Vet Microbiol. 109: 285-296.

[6]. Moodley A, Stegger M, Bagcigil AF, Baptiste KE, Loeffler A, Lloyd DH, Williams NJ, Leonard N, Abbott Y, Skov R, Guardabassi L. 2006. spa typing of Methicillin-resistant Staphylococcus aureus isolated from domestic animals and veterinary staff in the UK and Ireland. J Antimcrob Chemothr. 58:1118-1123.

[7]. Rankin S, Roberts S, O'Shea K, Maloney D, Lorenzo M, Benson CE. 2005. Panton-Valentine leukocidin (PVL) toxin positive MRSA strains isolated from animals. Vet Microbiol. 108:145-148.

[8]. Walther B, Wieler LH, Friedrich AW Hanssen A, Kohn B, Brunnberg L, Lübke-Becker A. 2008 Methicillin-resistant Staphylococcus aureus (MRSA) isolated from small and exotic animals at a university hospital during routine microbiological examinations. Vet. Microbiol. 127:171-178.

[9]. Kitai S, Shimizu A, Kawano J, Sato E, Nakano C, Uji T, Kitagawa H. 2005. Characterization of Methicillin-resistant Staphylococcus aureus isolated from retail raw chicken meat in Japan. J Vet Med Sci. 67:107-110.

[10]. Rich M, Roberts L, Kearns A. 2005 Methicillin-resistant Staphylococcus aureus isolated from animals. Vet Microbiol. 105:313314.

[11]. Jensen EL, Miller CL. 2001. Staphylococcus infections in Broiler Breeders.AviaTech.1(1). http://www.canadianpoultry.ca/cms_pdfs/AviaTech_Staph.pdf

[12]. Capita R, Alonso-Calleja C, Garcia-Fernandez MC, Moreno B. 2002. Characterization of Staphylococcus aureus isolated from poultry meat in Spain. Poultry Science 81:414-421.

[13]. Kohler B, Nattermann H, Witte W, Friedrichs F, Kunter E. 1980. Staphylococcus aureus infections in chickens in industrialized poultry units. 2. Microbiological studies: Staphylococcus aureus and other pathogens Arch Exp Veterinermed 34: 905-923.

[14]. Waters AE, Contente-Cuomo T, Buchhagen J, Liu CM, Watson L, Pearce K, Foster JT, Bowers J, Driebe EM, Engelthaler DM, Keim PS, Price LB. 2011. Multidrug-resistant Staphylococcus aureus in US meat and poultry. Clin Infect Dis. 52:1227-1230. doi: $10.1093 / \mathrm{cid} / \mathrm{cir} 181$

[15]. Pu S, Han F, Ge B. 2009. Isolation and characterization of Methicillin-resistant Staphylococcus aureus strains from Louisiana retails meats. Appl. Environ Microbiol. 75: 265-267.

[16]. Graham JP, Evans SL, Price LB, Silbsergeld EK. 2009. Fate of antimicrobial-resistant enterococci and staphylococcus and resistance determinants in stored poultry litter. Env Res. 109:682-689.

[17]. Buckley D. 2010. Diseases caused by chickens. http://www.livestrong.com/article/91095-diseases-caused-chickens/ Mar 23.

[18]. Wickline, S. 2013. Workers at livestock factories face increased MRSA Risk.

[19]. http://www.medpagetoday.com/InfectiousDisease/GeneralInfectiousDisease/40286. MedPage Today, Jul 5: 2013. 